\title{
ANÁLISE DE COMPONENTES PRINCIPAIS E CORRELAÇÕES ENTRE AS CARACTERÍSTICAS DO DOSSEL E DO SOLO EM PASTOS DE CAPIM-MARANDU
}

\author{
GALZERANO, Leandro ${ }^{1}$ \\ MORGADO, Eliane da Silva ${ }^{2}$ \\ RUGGIERI, Ana Claudia ${ }^{3}$ \\ MALHEIROS, Euclides Braga ${ }^{4}$
}

Recebido em: 2013-08-16

Aprovado em: 2014-04-13

ISSUE DOI: $10.3738 / 1982.2278 .962$

\begin{abstract}
RESUMO: Objetivou-se com este estudo avaliar as características do dossel e do solo por meio da análise de componentes principais durante o período de rebrotação do capim-marandu (Brachiaria brizantha cv. Marandu). O experimento foi conduzido na Universidade Estadual Paulista "Júlio de Mesquita Filho" - UNESP, Campus de Jaboticabal, SP. Para realização deste estudo foram tomados 15 pontos amostrais sobre o dossel do capim com 23 dias de rebrotação pós-corte, em junho de 2012. As variáveis avaliadas foram: altura do dossel, interceptação da radiação fotossinteticamente ativa (IRFA), índice de área foliar (IAF), emissão de $\mathrm{CO}_{2}$, temperatura e umidade do solo no mesmo ponto amostrado. Os resultados demonstraram que os dois primeiros componentes principais explicaram $75,73 \%$ da variação total. As variáveis altura, IRFA, IAF e umidade apresentaram associação positiva e foram correlacionadas negativamente com as variáveis do solo temperatura e $\mathrm{CO}_{2}$. A avaliação das características do dossel e do solo por meio da técnica de componentes principais demonstra que há associação positiva entre as características do dossel e a umidade do solo e entre a emissão de $\mathrm{CO}_{2}$ e a temperatura do solo. A emissão de $\mathrm{CO}_{2}$ do solo não é influenciada pelas características do dossel em pasto de capim-marandu.
\end{abstract}

Palavras-chave: Altura do dossel. Análise multivariada. Brachiaria. Emissão de $\mathrm{CO}_{2}$. Urochloa.

\section{PRINCIPAL COMPONENTS ANALYSIS AND CORRELATION OF CHARACTERISTICS OF THE CANOPY AND SOIL IN MARANDU PALISADEGRASS PASTURES}

\begin{abstract}
SUMMARY: The objective of this study was to evaluate the characteristics of canopy and soil through principal component analysis during the regrowth of marandu palisadegrass (Brachiaria brizantha cv. Marandu). The experiment was conducted at the Universidade Estadual Paulista "Júlio de Mesquita Filho" - UNESP, Jaboticabal, SP. For this study 15 sampling points were taken on the canopy of the grass with 23 days of regrowth after cutting, in June 2012. The variables evaluated were: canopy height, interception of photosynthetically active radiation (IPAR), leaf area index (LAI), $\mathrm{CO}_{2}$ emissions, soil temperature and moisture at the same point. The results showed that the first two principal components explained $75.73 \%$ of total variation. The variables height, IPAR, LAI and humidity presented positive association and were negatively correlated with the variables of soil temperature and $\mathrm{CO}_{2}$. The evaluation of the characteristics of canopy and soil using the technique of principal components shows that there is a positive association between the characteristics of canopy and soil moisture and between $\mathrm{CO}_{2}$ emission and soil temperature. The $\mathrm{CO}_{2}$ emission from the soil is not influenced by the characteristics of the canopy in marandu palisadegrass pastures.
\end{abstract}

Keywords: Canopy height. Multivariate analysis. Brachiaria. $\mathrm{CO}_{2}$ emission. Urochloa.

\footnotetext{
${ }^{1}$ Dr. em Zootecnia. Instituto Federal de Educação, Ciência e Tecnologia do Amazonas, Campus Manaus Zona Leste

${ }^{2}$ Pós-Doutoranda. Faculdade de Ciências Agrárias e Veterinárias, Universidade Estadual Paulista - Unesp.

Jaboticabal.

${ }^{3}$ Docente do Dpto de Zootecnia. Faculdade de Ciências Agrárias e Veterinárias, Universidade Estadual Paulista Unesp.

${ }^{4}$ Professor Titular do Depto. de Ciências Exatas da UNESP de Jaboticabal
} 


\section{INTRODUÇÃO}

As principais características da vegetação que recobre o solo são a altura do dossel, o índice de área foliar (IAF) e a interceptação da radiação fotosinteticamente ativa (IRFA) que geralmente estão correlacionadas entre si. A cobertura vegetal pode afetar a quantidade de radiação solar que chega ao solo, interferindo na umidade e temperatura do mesmo. O balanço de carbono no solo é dependente da relação existente entre o carbono fotossintetizado pelas plantas e as perdas de carbono para a atmosfera resultantes da oxidação microbiana do carbono orgânico a $\mathrm{CO}_{2}$, essa emissão é dependente principalmente da quantidade de matéria orgânica e da disponibilidade de resíduos vegetais no solo (COSTA et al., 2008). Vários fatores podem influenciar na emissão de $\mathrm{CO}_{2}$ do solo como umidade e temperatura deste, que interferem na atividade dos microorganismos do solo e, portanto, do $\mathrm{CO}_{2}$ emitido pela respiração e degradação de matéria orgânica além daquele emitido pela respiração das raízes das plantas. As técnicas de análise multivariada, não têm sido frequentemente utilizadas em pesquisas com forragens, no entanto, Da Silva e Sbrissia (2010), verificaram que os resultados obtidos pela análise de componentes principais demonstrou potencialidade de seu uso na interpretação de dados de pesquisa com plantas forrageiras, sendo esses resultados semelhantes aos obtidos com técnicas univariadas convencionais, porém com a vantagem na redução da dimensionalidade do conjunto de dados, retendo tanta informação quanto possível num menor número de componentes principais. Diante do exposto, objetivou-se com este trabalho estudar por meio de componentes principais e correlações, as relações entre a as características do dossel (altura, IAF e IRFA) e do solo (umidade, temperatura e emissão de $\mathrm{CO}_{2}$ ) em pastos de capim-marandu.

\section{MATERIAL E MÉTODOS}

O experimento foi conduzido na Universidade Estadual Paulista "Júlio de Mesquita Filho" - UNESP, Campus de Jaboticabal, SP, localizado a $21^{\circ} 15^{\prime} 22^{\prime \prime}$ ' de latitude Sul, e de $48^{\circ} 18^{\prime} 58^{\prime \prime}$ de longitude Oeste, a uma altitude de $595 \mathrm{~m}$. O solo da área experimental foi classificado como Latossolo Vermelho (Embrapa, 2006). Estudou-se o capim-marandu (Brachiaria brizantha, cv. Marandu), em uma área experimental de aproximadamente $5000 \mathrm{~m}^{2}$. O capim foi mantido sob cortes com roçadeira mecânica a $10,0 \mathrm{~cm}$ de altura desde agosto de 2011. Os cortes foram realizados quando o capim alcançava $40,0 \mathrm{~cm}$ de altura do dossel. Para realização deste estudo não foi realizada adubação e uma análise da camada arável do solo realizada em novembro de 2011, indicou as seguintes propriedades químicas: Ca: 43; $\mathrm{Mg}: 13$; $\mathrm{K}: 1,7 \mathrm{mmol} / \mathrm{dm}^{3}$; P (resina): $25 \mathrm{mg} / \mathrm{dm}^{3}$; MO: $20 \mathrm{~g} / \mathrm{dm}^{3} ; \mathrm{pH}\left(\mathrm{CaCl}_{2}\right): 5,9$. O clima de Jaboticabal, de acordo com a 
classificação de Köppen é classificado como tropical do tipo Aw, mesotérmico com verão úmido e inverno seco. Os dados agrometeorológicos registrados durante o período experimental, referentes à temperatura do ar, precipitação pluviométrica, número de dias com chuva e insolação foram obtidos junto a Estação de Agrometeorologia da Unesp, Campus de Jaboticabal, distante aproximadamente $1,0 \mathrm{~km}$ da área experimental (Tabela 1).

Tabela 1. Médias de temperatura média do ar, precipitação pluvial, número de dias com chuva e insolação durante o período experimental

\begin{tabular}{lcccc}
\hline Mês & $\begin{array}{c}\text { Temperatura média } \\
\text { do ar }\left({ }^{\circ} \mathrm{C}\right)\end{array}$ & $\begin{array}{c}\text { Precipitação } \\
\text { pluvial }(\mathrm{mm})\end{array}$ & $\begin{array}{c}\text { Número de dias } \\
\text { com chuva }\end{array}$ & $\begin{array}{c}\text { Insolação (h.mês- } \\
1 \text { ) }\end{array}$ \\
\hline Abril & $\mathbf{2 3 , 2}$ & $\mathbf{8 5 , 5}$ & $\mathbf{7}$ & $\mathbf{2 1 6 , 4}$ \\
Maio & $\mathbf{1 9 , 4}$ & $\mathbf{7 3 , 0}$ & $\mathbf{7}$ & $\mathbf{2 2 1 , 9}$ \\
Junho & $\mathbf{1 9 , 4}$ & $\mathbf{1 3 9 , 2}$ & $\mathbf{1 0}$ & $\mathbf{1 8 2 , 6}$ \\
Julho & $\mathbf{1 9 , 0}$ & $\mathbf{1 3 , 7}$ & $\mathbf{4}$ & $\mathbf{2 6 5 , 8}$ \\
\hline
\end{tabular}

Fonte: Estação de Agrometeorologia da Unesp, Campus de Jaboticabal.

Para realização deste estudo foram tomados 15 pontos amostrais sobre o dossel do capim em junho de 2012 quando o capim encontrava-se com 23 dias de rebrotação pós-corte, momento em que foi constatada grande heterogeneidade espacial em relação a altura do dossel. As características do dossel avaliadas foram altura do dossel, índice de área foliar (IAF) e interceptação da radiação fotossinteticamente ativa (IRFA) e as características do solo avaliadas foram umidade, temperatura e emissão de $\mathrm{CO}_{2}$. Todas as características estudadas foram tomadas no mesmo ponto amostrado. A altura do dossel foi determinada com uso de régua com graduação em centímetros, a IRFA e o IAF foram aferidos com o analisador de dossel, com sensor específico (AccuPAR Model LP - 80 PAR/LAI ceptometer) para esta faixa espectral da radiação solar (400 - $700 \mathrm{~nm}$ ). A emissão de $\mathrm{CO}_{2}$ do solo foi registrada utilizando-se uma câmara de fluxo (LI-COR LI-8100). A câmara de solos foi instalada sobre um colar de PVC, colocado no solo pelo menos $24 \mathrm{~h}$ antes da medição em cada ponto amostral próximo a esses pontos foram tomadas as medidas de umidade e temperatura do solo no período entre 9 e 10 horas da manhã, sendo utilizado termômetro de solo e medidor de umidade de solo digitais. Os colares de PVC são utilizados com objetivo de evitar distúrbios com a inserção da câmara diretamente no solo, o que acarretaria em aumento da emissão de $\mathrm{CO}_{2}$ superestimando o valor naquele ponto (PANOSSO et. al., 2008).

Os dados foram submetidos a análise de estatística multivariada utilizando a técnica de componentes principais e correlação simples utilizando-se o software Statistica (Statsoft 7.0). 


\section{RESULTADOS E DISCUSSÃO}

Na Tabela 2 estão apresentados os resultados da análise dos componentes principais entre as características do dossel e do solo e os componentes principais, em pasto de capim-marandu.

Tabela 2. Componentes principais $(\mathrm{CP})$, autovalores $\left(\Lambda_{\mathrm{i}}\right)$ e porcentagem da variância explicada pelos componentes (\%VCP) das características do dossel e do solo em pasto de capim-marandu

\begin{tabular}{lccc}
\hline Componente Principal & $K$ i & \%VCP & \%VCP (acumulada) \\
\hline CP1 & $\mathbf{3 , 2 9}$ & 54,93 & 54,93 \\
CP2 & $\mathbf{1 , 2 5}$ & 20,80 & 75,73 \\
CP3 & 0,72 & 11,97 & 87,70 \\
CP4 & 0,46 & 7,69 & 95,39 \\
CP5 & 0,21 & 3,47 & 98,86 \\
CP6 & 0,07 & 1,14 & 100,00 \\
\hline
\end{tabular}

Valores em negrito indicam os autovalores superiores a 1.

A análise dos componentes principais demonstrou que os dois primeiros componentes principais explicaram $75,73 \%$ da variação total, segundo o critério de Kaiser (1958), onde autovalores acima de 1 geram componentes com quantidade relevante de informações das variáveis originais.

Na Tabela 3 estão apresentados os coeficientes de correlação entre as variáveis avaliadas e os componentes principais. No CP1 pode ser observado que as variáveis altura, IRFA, IAF e umidade estão associadas positivamente, e correlacionadas negativamente com as variáveis do solo temperatura e $\mathrm{CO}_{2}$. Fato este explicável pois quanto maior a altura do pasto maior será a proporção de cobertura do solo pela forrageira, ou seja, maior será o índice de área foliar (IAF), o que garante maior proteção do solo as radiações solares, e dessa forma maior será a interceptação de radiação fotossinteticamente ativa (IRFA) mantendo assim maior umidade do solo. A maior temperatura do solo pode ser promovida pela menor cobertura do solo pela forrageira o que proporciona menor proteção do solo e como consequência menor umidade deste. $\mathrm{O}$ dossel forrageiro está constantemente em crescimento e desenvolvimento, mesmo que lento e, portanto, são dinâmicas as mudanças quanto a quantidade de luz no seu interior e também nas mudanças de temperatura e umidade do solo.

Tabela 3. Coeficiente de correlação entre variáveis (características do dossel e do solo) e os componentes principais, em pasto de capim-marandu

\begin{tabular}{lcccccc}
\hline \multirow{2}{*}{ Variável } & \multicolumn{7}{c}{ Componentes principais } \\
\cline { 2 - 7 } & CP1 & CP2 & CP3 & CP4 & CP5 & CP6 \\
\hline Altura & $-0,81$ & 0,43 & $-0,20$ & $-0,06$ & 0,35 & 0,02 \\
IRFA & $-0,94$ & $-0,05$ & 0,01 & $-0,22$ & $-0,17$ & 0,18 \\
IAF & $-0,94$ & 0,02 & $-0,13$ & $-0,21$ & $-0,15$ & $-0,18$ \\
Umidade & $-0,78$ & 0,16 & 0,12 & 0,58 & $-0,08$ & $-0,001$ \\
Temperatura & 0,48 & 0,62 & $-0,59$ & 0,06 & $-0,15$ & 0,03 \\
$\mathrm{CO}_{2}$ & 0,18 & 0,80 & 0,54 & $-0,14$ & $-0,06$ & $-0,01$ \\
\hline
\end{tabular}


No CP2 que explicou 20,80\% da variação total dos dados, foi verificada associação positiva entre as variáveis temperatura e $\mathrm{CO}_{2}$. A relação da emissão de $\mathrm{CO}_{2}$ com a temperatura tem sido observada na literatura por Costa et al. (2008) que inferiram que a emissão de $\mathrm{CO}_{2}$ possui variação sazonal e está correlacionadas positivamente com a temperatura do solo. Segundo Panosso et al. (2006) as variações diárias da emissão de $\mathrm{CO}_{2}$ do solo em épocas chuvosas são similares à temperatura do solo e não possuem relação com a ocorrência diária de chuva.

Na Tabela 4 estão apresentados os coeficientes de correlação simples entre as variáveis avaliadas no dossel forrageiro e no solo.

Tabela 4. Coeficientes de correlação simples entre as características do dossel e do solo avaliadas em pasto de capim-marandu

\begin{tabular}{lccccc}
\hline & Altura & IRFA & IAF & Umidade & Temperatura \\
\hline Altura & 1,00 & & & & \\
IRFA & $0,69^{*}$ & 1,00 & & & \\
IAF & $0,75^{*}$ & $0,92^{*}$ & 1,00 & & \\
Umidade & $0,61^{*}$ & $0,61^{*}$ & $0,61^{*}$ & 1,00 & \\
Temperatura & $-0,06$ & $-0,47^{* *}$ & $-0,36$ & $-0,30$ & 1,00 \\
$\mathbf{C O}_{2}$ & 0,08 & $-0,17$ & $-0,19$ & $-0,03$ & 0,27 \\
\hline
\end{tabular}

* Significativo a 5\% de probabilidade; ** Significativo a 10\% de probabilidade.

A altura do dossel é uma medida de fácil obtenção e a tentativa de relacioná-la com a IRFA pode facilitar atividades práticas de manejo das pastagens e como pode ser observado a IRFA apresentou alta correlação positiva (0,92\%) com o IAF. Brougham (1956) demonstrou que quando o nível de IRFA atinge 95\% da luz incidente, o IAF atinge um valor crítico, no qual a taxa de crescimento da cultura (TCC) estaria próximo de um valor máximo e o processo de rebrotação do pasto deve ser interrompido por corte o pastejo. No presente estudo, os dados de IRFA e IAF se relacionaram positivamente com a altura do dossel, corroborando os estudos já realizados correlacionando essas características em gramíneas forrageiras dos gêneros Cynodon, Panicum e Brachiaria (GALZERANO et al., 2012) e também em dosséis de leguminosas forrageiras (GALZERANO et al., 2013).

A emissão de $\mathrm{CO}_{2}$ do solo não apresentou correlação com as características do dossel avaliadas. Vários fatores podem interferir na emissão de $\mathrm{CO}_{2}$ do solo que possui variação sazonal sendo influenciada pela umidade, temperatura, atividade dos microorganismos e teor de matéria orgânica do solo, além da respiração das raízes das plantas, tipo de manejo do solo, dentre outros (COSTA et al., 2008; PANOSSO et al., 2009). 


\section{CONCLUSÃO}

A avaliação das características do dossel e do solo por meio da técnica de componentes principais demonstra que há associação positiva entre as características do dossel e a umidade do solo e entre a emissão de $\mathrm{CO}_{2}$ e a temperatura do solo. A emissão de $\mathrm{CO}_{2}$ do solo não é influenciada pelas características do dossel em pasto de capim-marandu.

\section{REREFÊNCIAS}

BROUGHAM, R.W. Effects of intensity of defoliation on regrowth of pasture. Australian Journal Agricultural Research. v. 7, p. 377-387, 1956.

COSTA, F.S.et al. Estoque de carbono orgânico no solo e emissões de dióxido de carbono influenciadas por sistemas de manejo no sul do Brasil. Revista Brasileira de Ciência do Solo, v. 32, p. 323-332, 2008.

DA SILVA, S.C.; SBRISSIA, A.F. Análise de componentes principais entre características morfogênicas e estruturais em capim-marandu sob lotação contínua. Ciência Rural, v.40, n.3, p.690-693, 2010.

\section{EMBRAPA - EMPRESA BRASILEIRA DE PESQUISA AGROPECUÁRIA. Sistema} Brasileiro de Classificação de Solos. Brasília: EMBRAPA, 2006. 306p.

GALZERANO, L.; MALHEIROS, E.B.; MORGADO, E.S.; RUGGIERI, A.C. Altura do dossel e interceptação de luz em dosséis de leguminosas forrageiras. Nucleus Animalium, v.5, n.1, p. 41-46, 2013.

GALZERANO, L.et al. Interceptação de luz e índice de área foliar em relação a altura do dossel de gramíneas forrageiras. Nucleus Animalium, v.4, n.2, p. 11-18, 2012.

KAISER, H.F. The varimax criterion for analytic rotation in factor analysis. Psychometrika, v.23, p.187-200, 1958.

PANOSSO, A.R. et al. Variabilidade espacial da emissão de CO2 em latossolos sob cultivo de cana-de-açúcar em diferentes sistemas de manejo. Engenharia Agrícola, v. 28, n. 2, p. 227-236, 2008 .

PANOSSO, A.R.et al. Emissão de $\mathrm{CO}_{2} \mathrm{em}$ um latossolo após preparo convencional e reduzido em períodos seco e chuvoso. Científica, v.34, n.2, p.257-262, 2006.

PANOSSO, A.R.et al. Variabilidade espacial da emissão de $\mathrm{CO}_{2}$, da temperatura e umidade de um latossolo desprovido de vegetação sob diferentes lâminas de molhamento. Semina: Ciências Agrárias, v. 30, p.1017-1034, 2009. 
